Research Article

\title{
Soil Contamination with Toxocara Eggs in Public Schools in Rural Areas of Southern Thailand
}

\author{
Nonthapan Phasuk (D), Ratee Kache, Kanjana Thongtup, Saowalee Boonmuang, \\ and Chuchard Punsawad
}

School of Medicine, Walailak University, Nakhon Si Thammarat, Thailand
Correspondence should be addressed to Chuchard Punsawad; chuchard.pu@wu.ac.th

Received 13 July 2020; Revised 17 August 2020; Accepted 26 August 2020; Published 8 September 2020

Academic Editor: Pedro P. Chieffi

Copyright (C) 2020 Nonthapan Phasuk et al. This is an open access article distributed under the Creative Commons Attribution License, which permits unrestricted use, distribution, and reproduction in any medium, provided the original work is properly cited.

\begin{abstract}
Soil is considered the primary source of Toxocara transmission to humans, especially children. The status of soil contamination with Toxocara eggs in southern Thailand is unknown. This study aimed at estimating the soil contamination with Toxocara eggs in public schools in Nakhon Si Thammarat province in southern Thailand. Soil samples were collected from 12 public schools between August and September 2017. At each site, ten soil samples were collected from the following five types of locations: (1) playgrounds, (2) football fields, (3) sidewalks, (4) schoolyards, and (5) areas around cafeterias. In total, 120 samples were examined for Toxocara eggs with a modified flotation method using a sucrose solution. Toxocara eggs were detected in 8 (66.7\%) of the 12 studied public schools. Of the 120 soil samples, 22 (18.3\%; 95\% CI: 11.9, 26.4) were contaminated with Toxocara eggs. The highest levels of Toxocara egg contamination were observed in playgrounds (41.7\%; 95\% CI: 22.1, 63.4), followed by football fields (20.8\%; 95\% CI: 7.1, 42.2), sidewalks (12.5\%; 95\% CI: 2.7, 32.4), and schoolyards (12.5\%; 95\% CI: 2.7, 32.4). There were significant differences in the distribution of Toxocara eggs across location types $(p<0.05)$. The findings demonstrated that the soil samples from public schools were contaminated with Toxocara eggs. Playgrounds were the most heavily contaminated locations. Teaching children proper handwashing steps and discouraging geophagia should be implemented to reduce the distribution of Toxocara and limit future Toxocara infections.
\end{abstract}

\section{Introduction}

Toxocariasis is a neglected parasitic infection caused by the larval stage of Toxocara canis (dog roundworm) and Toxocara cati (cat roundworm), which are intestinal parasites of dogs and cats, respectively [1]. Humans can be infected by accidentally ingesting embryonated Toxocara eggs shed by definitive hosts and through the consumption of third-stage (L3) larvae in raw or undercooked paratenic hosts $[2,3]$. Children have the highest risk of toxocariasis due to their behavior, such as eating soil, putting objects in their mouth, and eating earthworms; their close contact with infected dogs; and their poor hygiene habits $[4,5]$. The clinical manifestation of Toxocara infections in dogs is associated with the stage of infection. During larval migration through the lungs, dogs may develop a cough, nasal discharge, pneumonia, and pulmonary edema, while during adult worm infection, it may cause mucoid enteritis, vomiting, diarrhea, ascites, anorexia, and anemia [6]. Toxocara infections in humans can cause four primary clinical syndromes according to the organs affected: visceral larva migrans (VLM), a systemic disease caused by larval migration through major organs; ocular larva migrans (OLM), a disease limited to the eye and optic nerve; neurotoxocariasis (NT), a syndrome related to larval migration in the central nervous system that causes meningitis, encephalitis, cerebral vasculitis, and/or myelitis; and covert/ common toxocariasis, a nonspecific syndrome with nonspecific symptoms, wheezing, and eosinophilia [5, 7-10]. However, the vast majority of human Toxocara infections are asymptomatic, and serological testing using an enzymelinked immunosorbent assay has sufficient specificity to be 
the best indirect test for diagnosing this infection [7, 11]. A previous systematic review revealed that the estimated global Toxocara seroprevalence in humans was 19\% [12]. However, our recent study revealed that the seroprevalence of Toxocara canis infection in primary school children in rural southern Thailand was as high as 58\% [13].

The transmission of the Toxocara parasite occurs when an infected dog or cat sheds eggs in their feces in public parks or playgrounds. It takes 2-4 weeks for the larvae to develop and for the eggs to become infectious [4]. Consequently, soil contamination seems to be the most direct indicator of risk to human populations. A systematic review showed that the pooled global percentage of Toxocara egg contamination in public places was $21 \%$ [14]. Many previous studies carried out in different countries demonstrated that the contamination rate of Toxocara eggs ranged from 5 to $70 \%$ in soil in public parks, playgrounds, sandpits, beaches, backyards, sidewalks, and roadsides [8, 15-26]. Embryonated Toxocara eggs have been recovered from the hair of dogs $[27,28]$, which demonstrates that direct human-dog contact could also be a route of infection for humans. In southern Thailand, a previous study by Uga and colleagues revealed a $19 \%$ soil contamination rate with Toxocara eggs in Songkhla province [29]. However, the current status of soil contamination with Toxocara eggs in Thailand is still unclear and should be investigated further.

Currently, there are large numbers of stray dogs and cats in urban and rural areas of southern Thailand; these animals can easily access public areas. Furthermore, keeping animals as pets has increased in popularity. These factors may contribute to soil contamination with Toxocara eggs. Therefore, this study was designed to estimate the extent of soil contamination with Toxocara eggs in public schools in Nakhon Si Thammarat province in southern Thailand.

\section{Materials and Methods}

2.1. Study Area. Nopphitam is a district of Nakhon Si Thammarat province located in southern Thailand $\left(8^{\circ} 43^{\prime} 10^{\prime \prime} \mathrm{N}\right.$ latitude and $99^{\circ} 45^{\prime} 6^{\prime \prime} \mathrm{E}$ longitude) that has a total area of $720.2 \mathrm{~km}^{2}$ and had a population of 33,533 in 2019 . The climate in southern Thailand is considered to be tropical. In 2019, the Climatological Center, Thai Meteorological Department, reported that the average temperature in southern Thailand was $27.7^{\circ} \mathrm{C}$, with a minimum of $26.4^{\circ} \mathrm{C}$ in January and a maximum of $28.4^{\circ} \mathrm{C}$ in April. The annual rainfall was $1990 \mathrm{~mm}$. This district is divided into four subdistricts (tambons), including Nopphitam, Krung Ching, Karo, and Na Reng. Twelve public schools were located in Nopphitam district at the time of the study. The primary occupation is rubber and fruit plantation work, and the majority of the population is Buddhist.

2.2. Sample Collection. A total of 120 soil samples were collected from public schools in Nopphitam district between August and September 2017. At each school, ten soil samples were collected comprised of two samples from five different locations where children preferably played games or engaged in activities, including (1) playgrounds, (2) football fields, (3) sidewalks, (4) schoolyards, and (5) areas around cafeterias. The two samples from each location were randomly collected from two sites that were at least 20 meters apart, with $100 \mathrm{~g}$ of soil collected from a depth of $0-15 \mathrm{~cm}$ [26]. All samples were transferred into individually identified sterile plastic bags, sent to the Parasitological Laboratory of Walailak University, and stored at $4^{\circ} \mathrm{C}$ until analysis.

2.3. Detection of Toxocara Eggs. The soil samples were examined for Toxocara eggs with a modified flotation method using a sucrose solution $[26,30,31]$. In brief, the soil samples were dried overnight at room temperature and filtered through a $150 \mu \mathrm{m}$ mesh sieve. Approximately $2 \mathrm{~g}$ of powdered soil was transferred to a $15 \mathrm{~mL}$ conical tube (Corning, USA), suspended in approximately $8 \mathrm{~mL}$ of $0.05 \%$ Tween- 80 solution, and centrifuged at $2000 \mathrm{rpm}$ for 10 minutes. After discarding the supernatant, the test tube containing the sediment was filled to approximately $1 \mathrm{~cm}$ from the top with sucrose solution (specific gravity of 1.200), vortexed, and centrifuged again. The test tube was then filled to the very top with sucrose solution and centrifuged at $1800 \mathrm{rpm}$ for 5 minutes. A cover glass was added to the top of each tube and was examined for Toxocara eggs under a light microscope at a magnification of $400 \times$ by an experienced medical technologist.

2.4. Statistical Analysis. All statistical analyses were carried out using IBM SPSS Statistics for Windows, version 23.0. The rate of Toxocara contamination was assessed by determining the percentage with a 95\% confidence interval (95\% CI). Fisher's exact test was used to compare the rates of Toxocara contamination among the subdistricts and sites of specimen collection. A value of $p<0.05$ was considered significant.

\section{Results}

This study focused on Toxocara egg contamination in the soil of public schools in Nopphitam district using a modified flotation method with a sucrose solution. Our study revealed that Toxocara eggs were detected in $8(66.7 \%)$ of the 12 studied public schools in the rural area of Nakhon Si Thammarat province (Figure 1). The contamination rate in each of the subdistricts and public schools is shown in Table 1. Of the 120 soil samples, 22 (18.3\%; 95\% CI: 11.9, 26.4) soil samples were positive for Toxocara eggs. The highest level of soil contamination was observed in Karo subdistrict $(6 / 20,30 \%$; 95\% CI: $11.9,54.3)$, followed by Krung Ching subdistrict (8/30, 26.7\%; 95\% CI: 12.3, 45.9) and Nopphitam subdistrict (8/40, 20.0\%, 95\% CI: 9.1, 35.6) (Table 1). No Toxocara eggs were observed in $\mathrm{Na}$ Reng subdistrict. The overall differences in the contamination rates among the subdistricts were significant $(p<0.05)$ (Table 1).

According to the site of specimen collection, playgrounds were observed to have the highest Toxocara egg contamination rate $(41.7 \%$; $95 \%$ CI: $22.1,63.4)$, followed by 


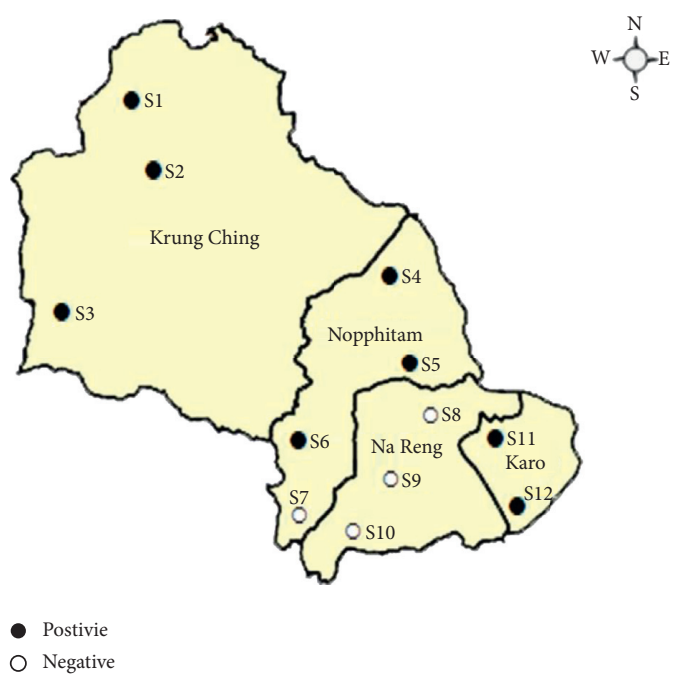

Figure 1: Map of Nopphitam district in Nakhon Si Thammarat province highlighting the distribution of positive (black circle) and negative (white circle) sites for Toxocara eggs in soil samples.

football fields (20.8\%; 95\% CI: 7.1, 42.2), sidewalks (12.5\%; 95\% CI: 2.7, 32.4), and schoolyards (12.5\%; 95\% CI: 2.7 , 32.4). The level of soil contamination was the lowest in areas around cafeterias $(4.2 \%$; 95\% CI: 1.0, 21.1) (Table 2). There were significant differences in the distribution of Toxocara eggs between the sites of specimen collection $(p<0.05)$.

\section{Discussion}

According to a previous epidemiological study, soil contamination by Toxocara eggs has been observed in many tropical and subtropical regions. To date, data on the contamination rate of Toxocara eggs in soil in the southern part of Thailand have been limited. The results of this study showed that 22 out of $120(18.3 \%)$ soil samples from public schools in rural southern Thailand were contaminated with Toxocara eggs, which was higher than those observed in previous studies conducted in other countries, such as $7 \%$ in various public places in Ardabil city in northwestern Iran [25], 4.75-12.84\% in public places in India [19, 20], and $14.9 \%$ in urban and rural areas of Poland [32]. The rate of contamination observed in our study was lower than those observed in other parts of the world, including $43 \%$ in public schools in the Philippines [17, 33], 28.6\% in public parks of Isfahan city in central Iran [21], $45 \%$ in public areas of Ahvaz in southwestern Iran [8], 53\% in public parks and playground sandpits in Greater Lisbon, Portugal [26], 20.4\% in children's play areas in southern England [34], 32-46\% in parks in northeastern Poland [23], and 45.8-54.5\% in urban and suburban areas of Malaysia [30]. However, the percentage of Toxocara egg contamination of soil determined in this study $(18.3 \%)$ was comparable to that observed in southeastern Asia (21\%) and the pooled global rate $(21 \%)$ reported in a systematic review [14]. In southern Thailand, a previous study conducted in the past decade reported that $19 \%$ of samples tested positive for Toxocara eggs in Songkhla province [29], a contamination rate that was similar to that observed in our study. Therefore, the reason for the discrepancy in the rate of contamination in previous studies may be due to several factors, including the culture, geographical parameters, climatic conditions, seasonal changes, soil types, type of cat and dog populations, people's attitudes toward pets, sample collection methods, examination methods, and diagnostic techniques [2, 24, 32, 35, 36].

Regarding the sites of specimen collection, the present study showed significant differences in the distribution of Toxocara eggs among the sites examined. The highest rate of Toxocara egg contamination was observed in playgrounds. Some studies have demonstrated that Toxocara eggs have been recovered in sand or soil samples from playgrounds in India [19, 20], Germany [24], and Turkey [37]. In southeastern Asian countries, a previous study conducted in the Philippines demonstrated that $42 \%$ of soil samples obtained from a public school were positive for Toxocara eggs, and a total of $49 \%$ of serum samples from children were positive for Toxocara infection [17]. There was a positive correlation between the Toxocara egg concentration and the seroprevalence of Toxocara infection [17]. A possible explanation for this observation may be that the number of stray dogs and cats has been increasing in rural areas, and they freely roam around school areas and defecate in playgrounds. Therefore, our study results suggest that the playgrounds may be the primary source of transmission of Toxocara eggs to humans, especially children. We suggest that schools should limit the access of dogs and cats to school areas, especially playgrounds. Schools should also teach children to avoid playing with soil and discourage geophagia to reduce the rate of Toxocara infection. These guidelines are essential for the development of effective programs aimed at minimizing the transmission of toxocariasis to children.

The present study was subjected to the following limitations. We did not collect data regarding other potential factors associated with the contamination rate, such as the details of the soil characteristics, climatic conditions, and seasonal changes. It has been reported that Toxocara eggs were most prevalent in low-acidity, relatively high-temperature, and high-moisture soil conditions [2, 3, 33]. Since we did not use molecular techniques in this study, we could not discriminate between Toxocara species since the eggs of both Toxocara species are similar in size and morphology. Molecular techniques are required for identifying the species of Toxocara eggs recovered in soil. Several previous reports have suggested that molecular methods are sufficiently sensitive to detect low levels of parasites and identify different Toxocara spp. [8, 16, 38, 39]. Since an assessment of the infection ability of Toxocara spp. eggs was not performed in this study, we could not calculate the overall viability rate of the eggs present in soil. This study reflects only the Toxocara egg contamination rates in public schools in rural areas; the contamination rates were not compared with those in urban areas. To determine the distribution of Toxocara eggs in different environments, further studies should investigate the presence of Toxocara eggs in soil samples collected from urban areas or different sampling areas, including public parks, beaches, and roadsides that dogs and cats can freely access. 
TABLE 1: Soil contamination by Toxocara eggs in the subdistricts and public schools in Nakhon Si Thammarat province in southern Thailand.

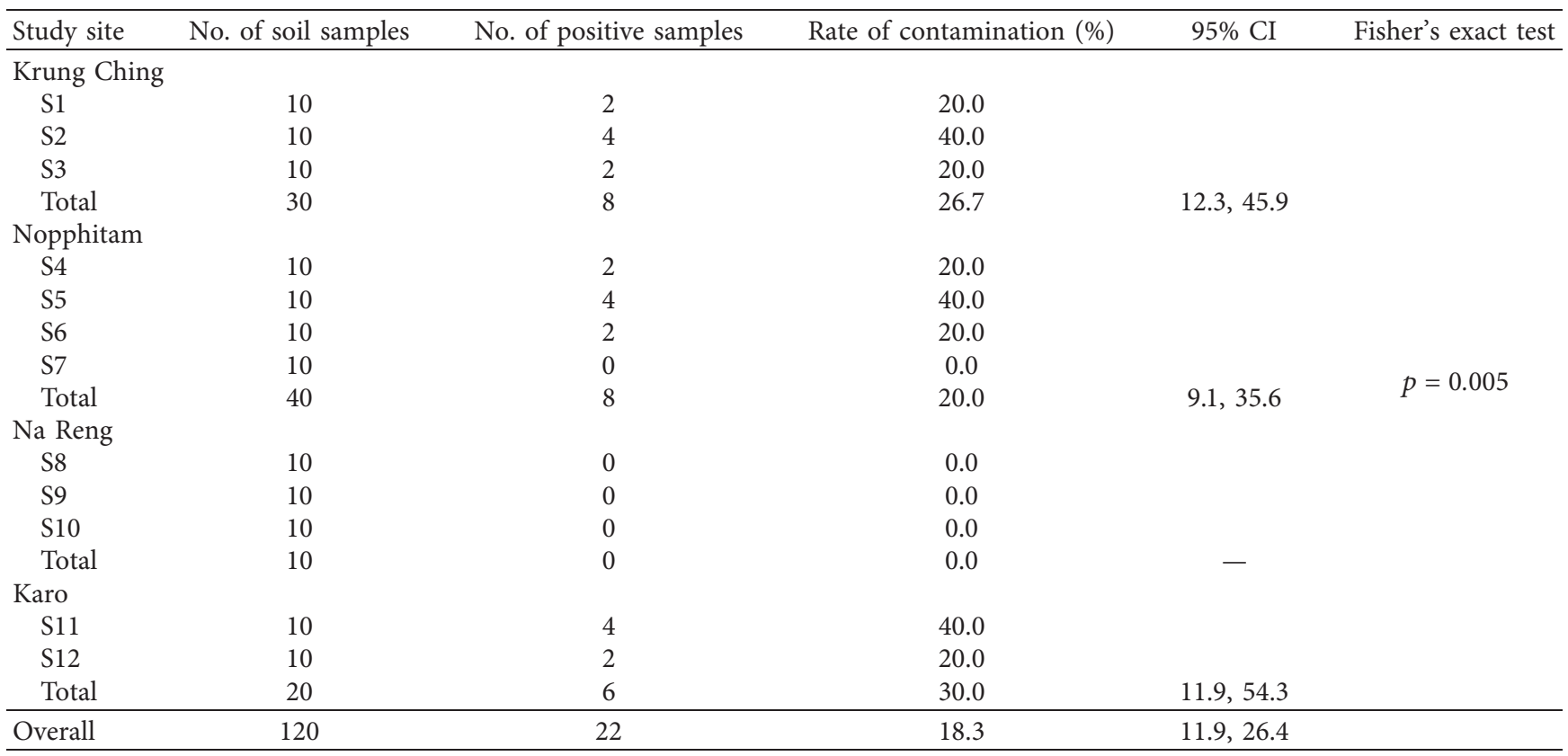

TABLE 2: Contamination rate of Toxocara eggs in the different sampling areas.

\begin{tabular}{|c|c|c|c|c|c|}
\hline Site of collection & No. of soil samples & No. of positive samples & Rate of contamination (\%) & $95 \% \mathrm{CI}$ & Fisher's exact test \\
\hline Playgrounds & 24 & 10 & 41.7 & $22.1,63.4$ & \\
\hline Football fields & 24 & 5 & 20.8 & $7.1,42.2$ & \\
\hline Sidewalks & 24 & 3 & 12.5 & $2.7,32.4$ & $p=0.016$ \\
\hline Schoolyards & 24 & 3 & 12.5 & $2.7,32.4$ & \\
\hline Areas around cafeterias & 24 & 1 & 4.2 & $1.0,21.1$ & \\
\hline Total & 120 & 22 & 18.3 & $11.9,26.4$ & \\
\hline
\end{tabular}

\section{Conclusions}

This investigation provides baseline knowledge regarding soil contamination with Toxocara eggs and important information regarding toxocariasis in southern Thailand. Playgrounds were the most heavily contaminated locations, indicating that playgrounds are a primary source of transmission of Toxocara eggs to children. Knowledge of areas contaminated with Toxocara eggs is essential for planning effective measures to prevent infection. Teaching children proper handwashing steps and not to eat soil should be implemented to reduce the distribution of Toxocara and prevent future Toxocara infections.

\section{Data Availability}

The data used to support the findings of this study are included in the article.

\section{Conflicts of Interest}

The authors declare that they have no conflicts of interest.

\section{Authors' Contributions}

N.P., R.K., and C.P. conceptualized the study, validated the data, and visualized the study and were involved in project administration. N.P., R.K., K.T., S.B., and C.P. carried out formal analysis. R.K., K.T., and S.B. investigated the study and were responsible for resources. N.P., R.K., K.T., and S.B. were involved in data curation. N.P. and C.P. prepared the original draft and reviewed and edited the manuscript. C.P. was responsible for supervision and funding acquisition. All authors have read and agreed to the published version of the manuscript.

\section{Acknowledgments}

The authors wish to thank the directors of all of the primary schools in Nopphitam district in Nakhon Si Thammarat province for their support throughout this study. The authors would like to acknowledge Dr. Prachyapan Petchuay, $\mathrm{MD}, \mathrm{PhD}$, Dean of the School of Medicine, Walailak University, for support throughout the course of this study. This research was partially supported by the New Strategic Research (P2P) Project of Walailak University, Thailand. 


\section{References}

[1] Centers for Disease Control (CDC), Toxocariasis, Centers for Disease Control (CDC), Atlanta, GA, USA, 2019.

[2] C. N. Macpherson, "The epidemiology and public health importance of toxocariasis: a zoonosis of global importance," International Journal for Parasitology, vol. 43, no. 12-13, pp. 999-1008, 2013.

[3] D. Traversa, A. Frangipane di Regalbono, A. Di Cesare et al., "Environmental contamination by canine geohelminths," Parasit Vectors, vol. 7, p. 67, 2014.

[4] D. M. Woodhall, M. L. Eberhard, and M. E. Parise, "Neglected Parasitic Infections in the United States: Toxocariasis," The American Journal of Tropical Medicine and Hygiene, vol. 90, no. 5, pp. 810-813, 2014.

[5] D. M. Woodhall and A. E. Fiore, "Toxocariasis: A Review for Pediatricians," Journal of the Pediatric Infectious Diseases Society, vol. 3, no. 2, pp. 154-159, 2014.

[6] A. Raza, J. Rand, A. G. Qamar et al., "Gastrointestinal Parasites in Shelter Dogs: Occurrence, Pathology, Treatment and Risk to Shelter Workers," Animals (Basel), vol. 8, no. 7, 2018.

[7] D. Despommier, "Toxocariasis: clinical aspects, epidemiology, medical ecology, and molecular aspects," Clinical Microbiology Reviews, vol. 16, no. 2, pp. 265-272, 2003.

[8] S. Khademvatan, F. Rahim, M. Tavalla et al., "PCR-based molecular characterization of Toxocara spp. using feces of stray cats: a study from Southwest Iran," PLoS One, vol. 8, no. 6, Article ID e65293, 2013.

[9] I. Mohammadzadeh, S. M. Riahi, V. Saber et al., "The relationship between Toxocara species seropositivity and allergic skin disorders: a systematic review and meta-analysis," Transactions of the Royal Society of Tropical Medicine and Hygiene, vol. 112, no. 12, pp. 529-537, 2018.

[10] A. Rostami, G. Ma, T. Wang et al., "Human toxocariasis-A look at a neglected disease through an epidemiological "prism"," Infection, Genetics and Evolution, vol. 74, Article ID 104002, 2019.

[11] J. Chen, Q. Liu, G. H. Liu et al., "Toxocariasis: a silent threat with a progressive public health impact," Infectious Diseases of Poverty, vol. 7, no. 1, p. 59, 2018.

[12] A. Rostami, S. M. Riahi, C. V. Holland et al., "Seroprevalence estimates for toxocariasis in people worldwide: A systematic review and meta-analysis," PLOS Neglected Tropical Diseases, vol. 13, no. 12, Article ID e0007809, 2019.

[13] N. Phasuk and C. Punsawad, "Seroprevalence of Toxocara canis infection and associated risk factors among primary schoolchildren in rural Southern Thailand," Tropical Medicine and Health, vol. 48, p. 23, 2020.

[14] Y. Fakhri, R. B. Gasser, A. Rostami et al., "Toxocara eggs in public places worldwide-a systematic review and metaanalysis," Environmental Pollution, vol. 242, pp. 1467-1475, 2018, Pt B.

[15] H. Bojar and T. Klapec, "Contamination of soil with eggs of geohelminths in recreational areas in the Lublin region of Poland," The Annals of Agricultural and Environmental Medicine, vol. 19, no. 2, pp. 267-270, 2012.

[16] J.-F. Durant, L. M. Irenge, R. Fogt-Wyrwas et al., "Duplex quantitative real-time PCR assay for the detection and discrimination of the eggs of Toxocara canis and Toxocara cati (Nematoda, Ascaridoidea) in soil and fecal samples," Parasites \& Vectors, vol. 5, no. 1, p. 288, 2012.

[17] A. J. Fajutag and V. G. Paller, "Toxocara egg soil contamination and its seroprevalence among public school children in Los Banos, Laguna, Philippines," The Southeast Asian Journal of Tropical Medicine and Public Health, vol. 44, no. 4, pp. 551-560, 2013.

[18] S. Horiuchi, V. G. Paller, and S. Uga, "Soil contamination by parasite eggs in rural village in the Philippines," Tropical Biomedicine, vol. 30, no. 3, pp. 495-503, 2013.

[19] N. R. Sudhakar, S. Samanta, S. Sahu et al., "Prevalence of Toxocara species eggs in soil samples of public health importance in and around Bareilly, Uttar Pradesh, India," Veterinary World, vol. 6, no. 2, pp. 87-90, 2013.

[20] D. Thomas and N. Jeyathilakan, "Detection of Toxocara eggs in contaminated soil from various public places of Chennai city and detailed correlation with literature," Journal of Parasitic Diseases, vol. 38, no. 2, pp. 174-180, 2014.

[21] M. Ghomashlooyan, M. Falahati, M. A. Mohaghegh et al., "Soil contamination with Toxocara spp. eggs in the public parks of Isfahan City, Central Iran," Asian Pacific Journal of Tropical Disease, vol. 5, no. Supplement 1, pp. S93-S95, 2015.

[22] A. Kleine, E. Janecek, P. Waindok, and C. Strube, "Flotation and adherence characteristics of Toxocara canis and T. cat $i$ and a reliable method for recovering Toxocara eggs from soil," Veterinary Parasitology, vol. 227, pp. 35-41, 2016.

[23] A. Kroten, K. Toczylowski, B. Kiziewicz, E. Oldak, and A. Sulik, "Environmental contamination with Toxocara eggs and seroprevalence of toxocariasis in children of Northeastern Poland," Parasitology Research, vol. 115, no. 1, pp. 205-209, 2016.

[24] A. Kleine, A. Springer, and C. Strube, "Seasonal variation in the prevalence of Toxocara eggs on children's playgrounds in the city of Hanover, Germany," Parasites \& Vectors, vol. 10, no. 1, p. 248, 2017.

[25] A. Pezeshki, A. Haniloo, A. Alejafar et al., "Detection of Toxocara spp. Eggs in the Soil of Public Places in and Around of Ardabil City, Northwestern Iran," Iranian Journal of Parasitology, vol. 12, no. 1, pp. 136-142, 2017.

[26] D. Otero, A. M. Alho, R. Nijsse, J. Roelfsema, P. Overgaauw, and L. Madeira de Carvalho, "Environmental contamination with Toxocara spp. eggs in public parks and playground sandpits of Greater Lisbon, Portugal," Journal of Infection and Public Health, vol. 11, no. 1, pp. 94-98, 2018.

[27] H. L. Amaral, G. L. Rassier, M. S. Pepe et al., "Presence of Toxocara canis eggs on the hair of dogs: a risk factor for Visceral Larva Migrans," Veterinary Parasitology, vol. 174, no. 1-2, pp. 115-118, 2010.

[28] B. Paoletti, D. Traversa, R. Iorio et al., "Zoonotic parasites in feces and fur of stray and private dogs from Italy," Parasitology Research, vol. 114, no. 6, pp. 2135-2141, 2015.

[29] S. Uga, W. Nagnaen, and V. Chongsuvivatwong, "Contamination of soil with parasite eggs and oocysts in Southern Thailand," The Southeast Asian Journal of Tropical Medicine and Public Health, vol. 28, no. Suppl 3, pp. 14-17, 1997.

[30] A. G. Loh and D. A. Israf, "Tests on the centrifugal flotation technique and its use in estimating the prevalence of Toxocara in soil samples from urban and suburban areas of Malaysia," Journal of Helminthology, vol. 72, no. 1, pp. 39-42, 1998.

[31] V. A. Santarém, L. P. Magoti, and T. D. Sichieri, "Influence of variables on centrifuge-flotation technique for recovery of Toxocara canis eggs from soil," Revista Do Instituto De Medicina Tropical De São Paulo, vol. 51, no. 3, pp. 163-167, 2009.

[32] H. Mizgajska-Wiktor, W. Jarosz, R. Fogt-Wyrwas, and A. Drzewiecka, "Distribution and dynamics of soil contamination with Toxocara canis and Toxocara cati eggs in Poland and prevention measures proposed after 20 years of study," Veterinary Parasitology, vol. 234, pp. 1-9, 2017. 
[33] V. G. Paller and E. R. De Chavez, "Toxocara (Nematoda: ascaridida) and other soil-transmitted helminth eggs contaminating soils in selected urban and rural areas in the Philippines," The Scientific World Journal, vol. 2014, Article ID 386232, 2014.

[34] R. Kirchheimer and D. E. Jacobs, "Toxocara species egg contamination of soil from children's play areas in Southern England," Veterinary Record, vol. 163, no. 13, pp. 394-395, 2008.

[35] D. Azam, O. M. Ukpai, A. Said, G. A. Abd-Allah, and E. R. Morgan, "Temperature and the development and survival of infective Toxocara canis larvae," Parasitology Research, vol. 110, no. 2, pp. 649-656, 2012.

[36] M. I. Gamboa, "Effects of temperature and humidity on the development of eggs of Toxocara canis under laboratory conditions," Journal of Helminthology, vol. 79, no. 4, pp. 327-331, 2005.

[37] F. S. Gurel, S. Ertug, and P. Okyay, "Prevalence of Toxocara spp. eggs in public parks of the city of Aydin, Turkey," Türkiye Parazitoloji Dergisi, vol. 29, no. 3, pp. 177-179, 2005.

[38] S. Khademvatan, R. Abdizadeh, and M. Tavalla, "Molecular characterization of Toxocara spp. from soil of public areas in Ahvaz Southwestern Iran," Acta Tropica, vol. 135, pp. 50-54, 2014.

[39] E. M. O’Connell and T. B. Nutman, "Molecular Diagnostics for Soil-Transmitted Helminths," The American Journal of Tropical Medicine and Hygiene, vol. 95, no. 3, pp. 508-513, 2016. 